\title{
Responsabilidad Social Universitaria: posicionamiento y pertinencia social de bachilleratos y universidades guanajuatenses
}

\author{
Norma Arriaga Villanueva* \\ Eva María Alonso Zaragoza** \\ Rosa Verónica Chacón PoncE*** \\ México
}

\section{Resumen}

El presente trabajo se concentra en identificar la forma como son percibidos bachilleratos y universidades en cuanto a su posicionamiento, nivel de recordación, costos, calidad, prestigio, presencia, criterios para seleccionar la universidad o bachillerato a estudiar y los hábitos de audiencia en medios de comunicación masiva y digital.

\section{Introducción}

Desde hace siglos las universidades son la base de la educación y del desarrollo de la sostenibilidad humana, su responsabilidad social es

* Doctor en Administración. Universidad Sabes, Centro Irapuato de México. Contacto: norma.arriagav@sabes.edu.mx

$\because$ Doctorante en Administración. Universidad Sabes, Centro Irapuato de México. Contacto: eva.alonso.z@sabes.edu.mx

*** Maestra en Mercadotecnia. Universidad Sabes, Centro Irapuato de México. Contacto: rosa.chaconp@sabes.edu.mx 
una cuestión relevante porque la preservación cultural de los recursos y el desarrollo sostenible de la sociedad se transfiere de una generación a otra (Thöni \& Schneller, 2011).

Con la misión de ofrecer educación en las comunidades urbanas y suburbanas del Estado de Guanajuato, el Sistema Avanzado de Bachillerato y Educación Superior (sabes) atiende de manera presencial a 37.000 jóvenes nivel preparatoria y 4.000 universitarios con educación presencial y a distancia, contribuyendo como agente educativo a la disminución de la marginalidad, migración, analfabetismo tecnológico e incrementando el índice de profesionistas y emprendedores que generen cambios productivos en su entorno; ya que, como afirma Carlos Monsiváis (2008), las universidades públicas representan el avance científico y cultural de una nación. Al respecto, se ilustra la fotografía 1 , en la cual se muestra la iniciativa emprendedora de alumnas de la Universidad Sabes Irapuato, quienes preservan nuestros valores culturales usando el chile cascabel, ingrediente muy usado en la gastronomía mexicana, para elaborar una nueva propuesta de galletas.

Fotografía 1. Alumnas universitarias con bases culturales elaboran galletas con tradicional ingrediente mexicano

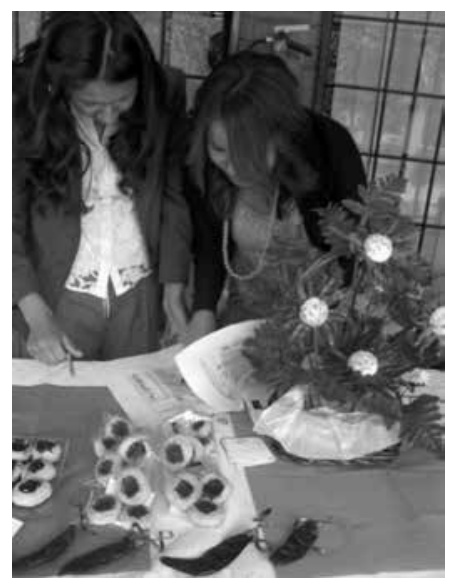

La Universidad es un actor clave del entretejido social que contribuye al desarrollo social, cultural y económico, por ello la pertinencia social se considera como eje de la Responsabilidad Social, porque se 
refiere a la medida en que la Universidad cumple las expectativas en el entorno social (Hernández-Arteaga, Alvarado-Pérez y Luna, 2015).

Es necesario mencionar que la Universidad está frente a dos imperativos que la mantienen en una tensión dinámica insuperable; por un lado, está identificada con la asimilación teórica y la respuesta histórica del conocimiento como origen y cimiento de la existencia; y, por otro lado, está obligada a transmitir el conocimiento a todos los educandos a través del proceso enseñanza-aprendizaje, la investigación y la Responsabilidad Social vivida en la comunidad universitaria y proyectada a la sociedad (Reinhard, Osburg y Towsend, 2010; Domínguez y Rama, 2012); de ahí que el papel de la Universidad consiste en asumir cada vez mejor su rol de formación superior integral con fines éticos y en pro del desarrollo sostenible de su comunidad (Vasilescu, Barna, Epure \& Baicu, 2010; Thöni \& Schneller, 2011; Ramos, García y Pérez, 2011; Beltrán-Llevador, Íñigo-Bajo \& Mata-Segreda, 2014).

De acuerdo con la Unesco (2009), la Educación Superior tiene la RS de hacer avanzar la comprensión de problemas con dimensiones sociales, económicas, científicas y culturales, así como la capacidad de hacerles frente. Desde este contexto, el compromiso de la Universidad exige de su habilidad y efectividad para responder a las necesidades de transformación de la sociedad donde está inmersa, mediante el ejercicio de sus funciones básicas, que son docencia, investigación y extensión y proyección social, esto significa que la Responsabilidad Social Universitaria se hace realidad cuando la organización toma conciencia de sí misma, de su entorno y de su papel en él (Beltrán-Llevador et al., 2014).

La Responsabilidad Social Universitaria emerge como un nuevo paradigma en el quehacer de las Instituciones de Educación Superior y su enfoque debe abarcar de manera transversal todas las funciones propias de las universidades que son docencia, investigación, extensión y gestión (Aristimuño, Rodríguez y Guaita, 2011; Ramos et al., 2011).

La incorporación e implementación de la Responsabilidad Social Universitaria cobra importancia debido al rol que juegan las IES como entes generadores de nuevos conocimientos y de profesionales que deben ser capaces de motorizar las transformaciones sociales, políticas y económicas que demanda la sociedad en búsqueda de lograr un desarrollo humano sostenible (Aristimuño et al., 2011). 
La fotografía 2 hace evidente el ímpetu de nuestros educandos como generadores de propuestas que contribuyen al desarrollo sustentable.

Fotografía 2. Alumno universitario realiza propuesta de riego que contribuye al cuidado del agua

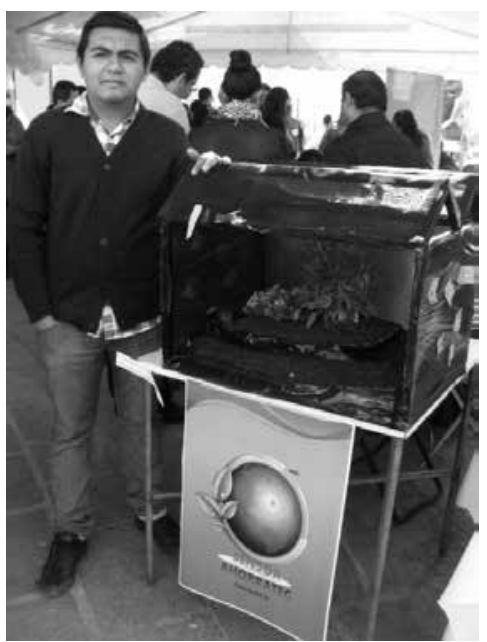

La gestión en las organizaciones no puede ser disgregada de su afectación al medio o contexto social donde operan, es así que se habla de su pertinencia y de los mecanismos distintos usados para mejorar su calidad, así como la gestión y rendir cuentas a la sociedad (Aristimuño et al., 2011).

Para la educación del futuro, los siete saberes sugieren replantear el papel de la educación para dar respuesta a los problemas globales y fundamentales de las sociedades, en el nivel medio superior y con relevancia en que las Instituciones de Educación Superior como instituciones sociales ejercen funciones estratégicas para el desarrollo cultural, científico y tecnológico y para los proyectos de consolidación de una sociedad. Al respecto, a continuación se proporcionan dos definiciones que tienen que ver con poder medir los resultados de las IES en su entorno o contexto interno y externo, obteniendo indicadores para medir si los objetivos propuestos se han cumplido o no (Aristimuño et al., 2011).

Para la Universidad Católica de Lima (2007), la Responsabilidad Social Universitaria es una política de calidad ética del desempeño de 
estudiantes, docentes y administrativos a través de la gestión responsable de los impactos educativos, cognitivos, laborales y ambientales que la universidad genera en un diálogo participativo con la sociedad para promover el Desarrollo Humano Sostenible (Aristimuño et al., 2011; Lescher, M., Lescher, I. \& Caira, 2015).

Para la Asociación de Universidades Jesuitas de América Latina (2007), la Responsabilidad Social Universitaria es la habilidad y efectividad de una universidad para responder a las necesidades de transformación de la sociedad donde está inmersa, mediante el ejercicio de sus funciones sustantivas, que son docencia, investigación, extensión y gestión. Estas funciones deben estar animadas por la búsqueda de la promoción de la justicia, solidaridad y equidad social mediante la construcción de respuestas exitosas para los retos que implica promover el Desarrollo Humano Sostenible (Aristimuño et al., 2011, p. 4).

Los indicadores de Responsabilidad Social Universitaria validados en estas definiciones son:

Educativo. Determina la presencia en el currículo de ejes, asignaturas y proyectos que reflejen participación activa en la prestación de servicios y soluciones a problemáticas sociales en las comunidades (Aristimuño et al., 2011).

Epistémico-cognitivo. Muestra la producción, generación de saberes, conocimientos, ciencia y apropiación de estos por la sociedad (Aristimuño et al., 2011).

Social. Relación de los actores sociales para a través de esta vinculación con el entorno solventar problemas de desarrollo social (Aristimuño et al., 2011).

Ambiental. Determina el conjunto de acciones que establece la incorporación de los aspectos medioambientales y sus impactos (Aristimuño et al., 2011).

Funcionamiento organizacional. Determina la relación de las Instituciones de Educación Superior con sus clientes y proveedores internos y externos (Aristimuño et al., 2011).

Este planteamiento muestra que al ser la Responsabilidad Social Universitaria una estrategia integrada de gestión debe ser medida de tal 
forma en que se puedan comprobar sus resultados y controlar su desarrollo. Los indicadores facilitan la medición del comportamiento de las variables en función de una meta planificada (Aristimuño et al., 2011).

$\mathrm{Al}$ desarrollar sus misiones de educación, investigación y dimensión social, las universidades son actores claves para el nuevo modelo de la sociedad basada en el conocimiento. Una revisión a la literatura internacional sobre pertinencia social universitaria permite constatar una atención creciente en su relación con la responsabilidad y la vinculación social. Al respecto, en los documentos especializados de la Unesco (1995), con ocasión del Encuentro Mundial sobre la Enseñanza Superior en el siglo veintiuno, el concepto de pertinencia social es reforzado sistemáticamente y definido como la capacidad de responder a las necesidades o problemas sociales (Beltrán-Llevador et al., 2014).

El concepto de pertinencia social emitido por la Organización de las naciones Unidad para la Educación, la Ciencia y la Cultura (1995) se enmarca en las nuevas formas de producción de conocimiento que debe surgir a partir del contexto de aplicación, así el conocimiento pertinente es a partir de las demandas y enfocan su interés en lo local y lo regional (Ramos et al., 2011; Beltrán-Llevador et al., 2014).

Puesto que el desarrollo económico de las regiones depende de los esfuerzos invertidos por las instituciones que producen y difunden nuevos conocimientos científicos y tecnológicos, entonces las universidades como tal tienen que orientarse al mundo de los negocios y crear más riqueza y bienestar social cuanto más se consiga aplicar los conocimientos. Dicho esto, es necesario hacer énfasis en el paradigma del poder transformador de la Universidad sobre la sociedad y es que a partir de la generación del conocimiento y de llevar el conocimiento a todo el que lo requiera, se puede impulsar la transformación como obligación ética (Beltrán-Llevador et al., 2014).

Lo anterior expuesto permite afirmar que la Universidad requiere definir la visión integral de sus funciones académicas: docencia, investigación y extensión sobre un nuevo paradigma como eje transformador que le permita, a través de la creación y difusión del conocimiento, lograr la correspondencia entre lo que la sociedad demanda y, por otra parte, la coherencia interna que debe prevalecer, así como en la búsqueda de la pertinencia social que aspira el contexto de las instituciones 
de educación superior, tratando de hacer compatible el discurso de la acción (Ramos et al., 2011; Beltrán-Llevador et al., 2014).

Respecto a su finalidad, la Responsabilidad Social Universitaria:

a. Orienta a las universidades hacia una clara conciencia de su misión, es decir; hacia una opción ético-política de contribución al desarrollo humano y sustentable, la equidad, a inclusión social, los derechos humanos y la cultura de la paz.

b. Posibilita a generar políticas y estrategias adecuadas que aseguren la congruencia de sus procesos de docencia, investigación, extensión y gestión adecuadas con dicha misión y a la cual direccione su capital humano, relacional, intelectual tecnológico y económico.

c. Propicia una adecuada concepción de la autonomía enfatizando la capacidad de tomar sus propias decisiones responsabilizándose de sus procesos e impactos y a la vez atendiendo a los requerimientos, interpelaciones y necesidades de todos.

d. Crea condiciones para una cultura de la observación y escucha propia y externa, que genere el diagnóstico, la evaluación de sus procesos, acciones e impactos presentes, tanto cognitivos, como educacionales, sociales y medioambientales.

e. Genera la trasparencia y la rendición de cuentas tanto a la comunidad universitaria y la sociedad en general a la que se debe.

f. Permite la integración curricular de la Responsabilidad Social en forma de modelo educativo, programas específicos de ética aplicada, así como procesos evolutivos propios o característicos, y de técnicas y metodologías pedagógicas adecuadas dispuestas para tal fin (Beltrán-Llevador et al., 2014).

Atendiendo a su función social, la Universidad forma profesionales de excelencia y al mismo tiempo personas éticamente comprometidas, de esta manera, cumple sus responsabilidades formando profesionistas con conciencia histórica y espíritu cívico, capaces de reconocer la situación de sus sociedades y de entender sus deberes hacia sus conciudadanos. Entre estas personas capaces y honradas deben surgir los líderes de una sociedad humanitaria, justa y además eficiente que actúen con 
honradez, respeto a la verdad y sentido crítico (Nejati, Shafaei, Salamzadeh \& Daraei, 2011; Domínguez y Rama, 2012).

La Responsabilidad Social Universitaria es, entonces, una nueva mega tendencia del sector educativo en la que se encuentran diversos países como Reino Unido, Finlandia, Alemania, Francia, México, Chile y Australia, que han comenzado a plantear nuevos retos, así mismo organismos internacionales como la Organización para la Cooperación y Desarrollo Económicos y la Organización de las Naciones Unidas para la Educación, la Ciencia y la Cultura, que llevan a cabo discusiones con la Comisión Europea formulando importantes postulados (Domínguez y Rama, 2012) y declaraciones como la del decenio de la Organización de las Naciones Unidas de la Educación para el Desarrollo Sostenible, que sostiene una oportunidad para IES para promover la formación y conciencia de un futuro sostenible (Mehta, 2011).

Lo anterior, hace necesario el uso de un modelo de Responsabilidad Social Universitaria que permita interactuar, activar procesos didácticos, pedagógicos y organizacionales, establecer relaciones con el entorno y con el gobierno para favorecer a la sociedad, estudiantes, profesores y a la universidad como tal (Nejati et al., 2011; Morales, 2013).

En sintonía con el movimiento de la Responsabilidad Social la Universidad ha empezado a concebir conceptos que respondan al qué, diseñar estrategias y al cómo, y orientar su servicio para responder al para qué (Domínguez y Rama, 2012).

El cuadro 1 permite comprender que la interrelación del rol, las funciones de la Universidad y Responsabilidad Social Universitaria son motor de la trasformación social, económica, científica y tecnológica con compromiso ético: también enfatiza que la Responsabilidad Social Universitaria garantiza la calidad de la educación superior en su conjunto al formar profesionales con conocimientos pertinentes a los requerimientos del entorno, sensibles y motivados por los valores, orientar la investigación científica a la solución de los problemas sociales y desarrollar proyectos con impacto social real, propiciar la transferencia del conocimiento y tecnología a la sociedad, capacitar a sus profesores y empleados al más alto nivel, concientizar a los alumnos sobre la corresponsabilidad de todos en la solución de los problemas mundiales (Reinhard, Osburg \& Towsend, 2010; Valarezo y Tuñez, 2014). 
Cuadro 1. Relación de Responsabilidad Social Universitaria con rol y funciones de la universidad, importancia de la imagen y posicionamiento

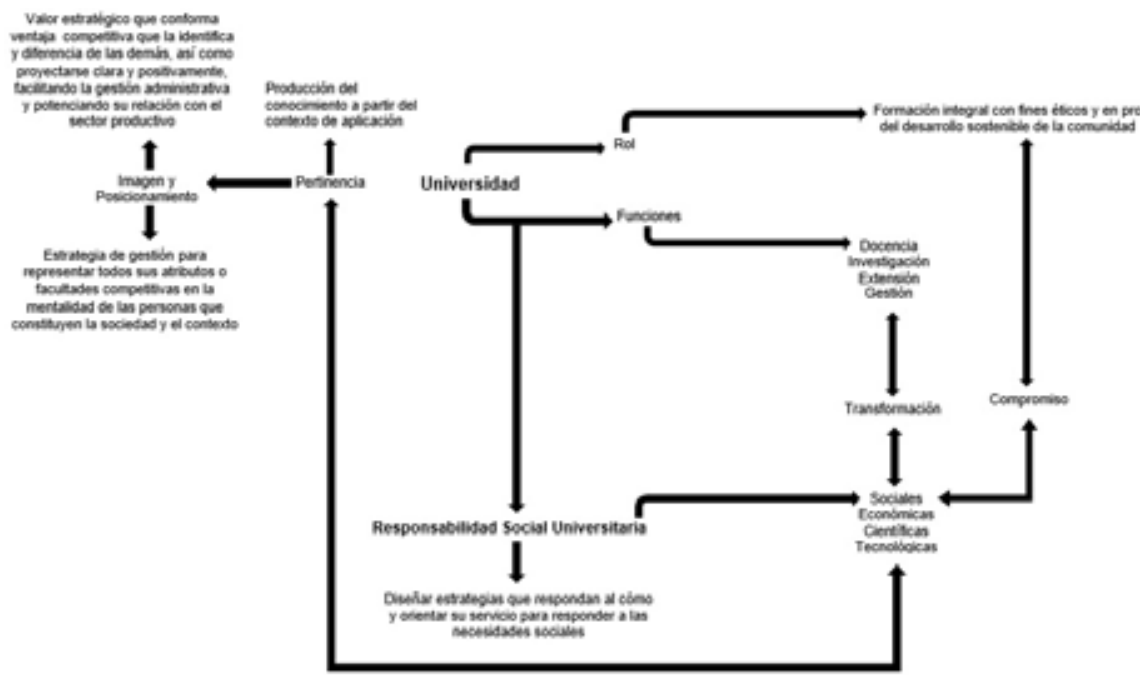

Fuente: elaboración propia.

Este valioso constructo que muestra el cuadro 1 fue diseñado a partir de la evidencia empírica existente, y permite relacionar la pertinencia con la imagen y posicionamiento, ya que constituyen una estrategia de gestión para las Instituciones de Educación Superior para representar todos sus atributos o facultades competitivas en la mentalidad de las personas que constituyen la sociedad y el contexto, así; la imagen es un activo a manera de propiedad de la organización que construye internamente la propia entidad y la comunica a sus públicos (Rivera y Martínez, 2014). Es necesario mencionar que divulgar información completa, precisa, adecuada a través del mejor canal disponible, permite a los beneficiarios la activación de una retroalimentación y consolidación del proceso con la toma de decisiones (Baraibar \& Luna, 2012).

La imagen otorga a las universidades conformar una ventaja competitiva que la identifica y diferencia de las demás, así como proyectarse clara y positivamente, facilitando la gestión académica y administrativa y potenciando su relación con el sector productivo, por ello la imagen se perfila como un valor estratégico cada vez más importante como consecuencia de estar inmersos en un entorno muy competitivo, en el 
que los clientes potenciales cada vez demandan y reciben más información (Rivera y Martínez, 2014).

La imagen corporativa de una Universidad es un concepto multidimensional basado en una variedad de factores, como el tamaño de la organización, su capacidad de gestión, el grado de implicación de los distintos públicos, las normas y las relaciones internas con el personal, la implicación de la institución con la sociedad en la que se inserta y sus responsabilidad social, la calidad percibida de sus productos y servicios o la comunicación institucional que genera (Rivera y Martínez, 2014).

El posicionamiento es un término acuñado en los años setenta y hoy un concepto ampliamente manejado en el ámbito de la comunicación, la investigación de mercados, siendo uno de los elementos clave en la gestión del marketing moderno; este se refiere a estrategias orientadas a crear y mantener en la mente de los clientes un determinado concepto del producto o servicio de la organización en relación con la competencia; y está basado en la percepción de los consumidores (Rivera y Martínez, 2014).

Ante el incremento competitivo característico del mercado universitario actual, uno de los aspectos que aportan un activo de considerable valor y muy ventajoso es la identificación de su propia marca corporativa y su posicionamiento óptimo entre sus principales públicos, por ello las Instituciones de Educación Superior procuran tener una imagen positiva y un posicionamiento en el sector productivo tanto en las relaciones de carácter formal como informal, debido a la incidencia de ambos aspectos: la imagen institucional y el posicionamiento de la Universidad en las decisiones con sus clientes, teniendo un efecto de retención y atracción (Rivera y Martínez, 2014).

Los especialistas en mercadotecnia se sirven de muchas formas de comunicación personal y no personal, para informar y persuadir a los mercados deseados. Elegir la combinación de medios eficaz es una importante tarea de mercadotecnia (Stanton, Etzel \& Walker, 2007) y han adoptado el concepto de una mezcla de marketing expandida; además de las cuatro "P”, incluye, además personas, evidencia física y proceso (Zeithmal, Bitner \& Gremler, 2009). 
La empresa debe satisfacer las necesidades del mercado meta y elaborar un programa de marketing completo para llegar a los consumidores utilizando la combinación producto, precio, plaza, promoción (Kerin, Hartley \& Redelius, 2009).

A continuación se presenta cómo son percibidos bachilleratos y universidades en cuanto a su posicionamiento, nivel de recordación, costos, calidad, prestigio, presencia, criterios para seleccionar la universidad o bachillerato a estudiar y los hábitos de audiencia en medios de comunicación masiva y digital.

Desde esta perspectiva, cabe formular la pregunta que guía el presente estudio: ¿cómo son percibidos bachilleratos y universidades en cuanto a su posicionamiento, nivel de recordación en México? Al respecto, el propósito de este trabajo se concentra en analizar la posición y percepción que ocupan los bachilleratos y las universidades en el mercado, en términos de costos, calidad, presencia en el estado, para decidir en qué institución estudiar.

\section{Metodología de investigación}

La investigación de mercados incluye la identificación, recopilación, análisis, difusión y uso de la información. Benasini (2009) menciona que la investigación de mercados es la reunión, el registro y el análisis de todos los hechos acerca de los problemas relacionados con las actividades de las personas, las empresas y las instituciones en general, en el caso de las organizaciones públicas, la investigación de mercados contribuye a una mejor comprensión del entorno que les permite tomar mejores decisiones de tipo económico, político y social.

Debido a factores como tiempo y costo, los parámetros de una población o proceso frecuentemente se estiman sobre la base de estadísticas muestrales. Un parámetro es un valor de resumen para una población o proceso, mientras que una estadística muestral es un valor de resumen para una muestra. Para emplear una estadística muestral como estimador de un parámetro, la muestra debe ser aleatoria, que es un tipo de muestra en el que todos los elementos de la población de interés o 
población objetivo tienen una oportunidad conocida, usualmente igual, de ser elegidos para una inclusión en la muestra (Kazmier, 1998).

Se propone un plan de muestreo para la obtención de información confiable de parte de sujetos de las ciudades de influencia de los bachilleratos y de las universidades. Estos estudiantes conforman la principal población a través de la cual las instituciones educativas pueden cumplir con la misión de formación a nivel medio superior y superior (Klinger et al., 2014, p. 83).

Cada estudio tiene un tamaño muestral idóneo, que permite comprobar lo que se pretende con la seguridad y precisión fijadas por el investigador. Esta seguridad y precisión dependerán del rango de posibles respuestas (necesitaremos una muestra más pequeña si las opciones son sí o no, que si por el contrario la opción es elegir entre 1 y 10). Así mismo, también influirá si el estudio se realiza sobre una población finita o infinita. Para el caso de este estudio se realiza en base a una población finita.

Para predecir el futuro con cierto grado de certeza esta investigación es concluyente descriptiva con diseño transversal múltiple (Malhotra, 2008). La muestra está representada por 1.605 personas, apoyándose del Censo Nacional 2010, Inegi y Censo del sector productivo localizado a través del Sistema de Información Empresarial Mexicano, bajo muestreo aleatorio por conglomerados. El instrumento se integró en escala de comparación, usando el Diseño y Análisis de Encuestas Versión 4 (Dyane V4).

La muestra que se toma para el estudio se aplica a personas desde los 11 hasta mayores de 50 años. Se utiliza el método de encuesta. El instrumento para realizar la recogida de datos está integrado por la técnica de escalamiento de opción múltiple, con 20 preguntas.

Para llevar a cabo esta investigación se toma como base el proceso de investigación de mercados propuesto por Malhotra (2008). Se considera que el proceso de investigación de mercados consta de seis pasos, según se muestra a continuación.

\section{Paso 1. Definición de la información necesaria}

Comprende la definición de criterios sobre indicadores que complemente el estudio sobre posicionamiento e imagen. Así mismo, definir y obtener 
información de censos del Inegi sobre edades por rangos (igual o mayor a 11,15 ; a 19,20 ; a 24,25 ; a 29,30 ; a 34,35 ; a 39,40 ; a 49 , mayor o igual a 50) de los municipios con mayor presencia del Sistema, entre los que se encuentran: Acámbaro, Apaseo el Grande, Celaya, Comonfort, San Felipe, Juventino Rosas, Irapuato, Pénjamo, Salvatierra, San José Iturbide, San Luis de la Paz, Villagrán. Igualmente, determinar la población por municipio en cuanto a sector educativo: secundaria, nivel medio superior, superior, así como la cantidad de empresas en los diversos municipios.

\section{Paso 2. Análisis de datos secundarios}

Estudio de posicionamiento realizado en 2013.

Censo Nacional del 2014 del Instituto Nacional de Estadística y Geografía.

Censo del sector productivo localizado a través del Sistema de Información Empresarial Mexicano.

\section{Paso 3. Técnicas para la obtención de datos cuantitativos}

Se utiliza la técnica de encuesta, que es un interrogatorio hacia los sujetos, a quienes se les plantea una variedad de preguntas. Para el diseño del instrumento se considera la aplicación de preguntas generales en cuanto a plantel, género, escolaridad y ocupación.

\section{Paso 4. Procedimiento de medición y escalamiento}

Se propuso caracterizar esta población mediante un instrumento denominado encuesta (Klinger et al., 2014, p. 83). El instrumento está integrado por la técnica de escalamiento en escala comparativa.

\section{Diseño del cuestionario}

Se determinó la población objeto de estudio (Klinger et al., 2014, p. $83)$. Se realiza un solo cuestionarios que se aplica a los tres sectores en donde se asignan preguntas generales. 


\section{Paso 5. Proceso de muestreo y tamaño de la muestra}

Se determinó la población de muestreo (Klinger et al., 2014, p. 83), para ello se aplica la fórmula probabilística para poblaciones homogéneas finitas.

La muestra que se toma es aleatoria, ya que es necesario que los elementos de la población objetivo tengan la misma oportunidad de inclusión en la muestra (Kazmier, 1998). Para el caso de este estudio, se realiza el estudio con base en una población finita. Se aplica la fórmula probabilística para poblaciones homogéneas finitas, los valores se muestran a continuación:

$$
n=\frac{z^{2} p q N}{e^{2}(N-1)+z^{2} p q}
$$

Z 1.96 nivel de confianza del $95 \%$

p 0.95 porción esperada $5 \%$

q 0.05 Probabilidad en contra

e 0.3 Precisión de $3.0 \%$

$\mathrm{N}$ Varía de acuerdo al sector. Los valores del universo para cada conglomerado son: sector población general, 4.276.672; sector educativo, 1932 y sector empresarial 30.450 .

Dada la naturaleza de esta investigación, se tienen tres universos diferentes: la población del sector educativo, empresarial y en general, entonces se obtienen tres muestras, según los muestran las tablas 1 a 3 .

La tabla 1 corresponde a la muestra de la población general con participación de 600 personas en rangos de edad de 11 a 50 años de los municipios de Acámbaro, Apaseo el Grande, Celaya, Comonfort, San Miguel de Allende, Dolores Hidalgo, San Felipe, Guanajuato, Juventino Rosas, Irapuato, León, Silao, Pénjamo, Salvatierra, San José Iturbide, San Luis de la Paz, Salamanca y Villagrán.

La Tabla 2 corresponde a la muestra de población del sector educativo con participación de 619 personas de las cuales 367corresponden al sector educativo de nivel secundaria, 195 de medio superior y 57 de nivel superior de los municipios de Acámbaro, Apaseo el Grande, 
Tabla 1. Muestra de población general

\begin{tabular}{|c|c|c|c|c|c|c|c|c|c|}
\hline \multirow{2}{*}{ Chedad } & \multicolumn{9}{|c|}{ Poblocidn en seneral } \\
\hline & $11-14$ allot & 15-19 allot & $20-24$ allot & $25-29$ allor & 30-34 ahos & 35-39 ahos & 40-49 ahlas & $\begin{array}{l}\text { Mayor f } \\
\text { isuala so }\end{array}$ & Sutotal \\
\hline Acambaro & 4 & 1 & 1 & 1 & 1 & 1 & 2 & 3 & 14 \\
\hline Apase o el Grande & 3 & 1 & 1 & 1 & 1 & 1 & 1 & 1 & 10 \\
\hline Celaya & 19 & 6 & 6 & 5 & 5 & 5 & 8 & 11 & 65 \\
\hline Comonfort & 4 & 1 & 1 & 1 & 1 & 1 & 1 & 1 & 11 \\
\hline San Mruel de Allende & 8 & 3 & 2 & 2 & 2 & 2 & 2 & 2 & 23 \\
\hline Dolores Midalgo & 7 & 2 & 2 & 2 & 1 & 1 & 2 & 3 & 20 \\
\hline San Felipe & 5 & 2 & 1 & 1 & 1 & 1 & 1 & 2 & 14 \\
\hline Quanajuato & 7 & 3 & 2 & 2 & 2 & 2 & 3 & 4 & 25 \\
\hline Juventino Rosas & 2 & 1 & 1 & 1 & 1 & 1 & 1 & 3 & 11 \\
\hline Irapuato & 23 & 8 & 7 & 6 & 6 & 6 & 8 & 12 & 76 \\
\hline Leon & 62 & 21 & 18 & 17 & 16 & 15 & 23 & 30 & 202 \\
\hline 5190 & 8 & 3 & 2 & 2 & 2 & 2 & 2 & 3 & 24 \\
\hline Pénjamo & 6 & 2 & 2 & 1 & 1 & 1 & 2 & 4 & 19 \\
\hline Salvatierra & 4 & 1 & 1 & 1 & 1 & 1 & 1 & 3 & 13 \\
\hline San Jose iturbide & 4 & 1 & 1 & 1 & 1 & 1 & 1 & 1 & 11 \\
\hline San Luls de la Paz & 6 & 2 & 2 & 1 & 1 & 1 & 2 & 1 & 16 \\
\hline Salamanca & 10 & 4 & 3 & 3 & 3 & 3 & 4 & 7 & 37 \\
\hline Villagrán & 2 & 1 & 1 & 1 & 1 & 1 & 1 & 1 & 9 \\
\hline
\end{tabular}

Fuente: elaboración propia.

Tabla 2. Muestra de población sector educativo

\begin{tabular}{|c|c|c|c|c|}
\hline \multirow[b]{2}{*}{ Ciudad } & \multicolumn{4}{|c|}{ Sector Educativo } \\
\hline & Secundanias & $\begin{array}{c}\text { Mledar } \\
\text { Superior }\end{array}$ & Superior & Subtotal \\
\hline Acámbato & 13 & 4 & 1 & 18 \\
\hline Apaseo el Grande & 10 & 3 & 0 & 13 \\
\hline Celara & 31 & 24 & 8 & 63 \\
\hline Comonfort & 9 & 4 & 1 & 14 \\
\hline San Maguel de Allende & 21 & 9 & 7 & 37 \\
\hline Dolores Hidalgo & 24 & 7 & 1 & 32 \\
\hline San Felipe & 21 & 5 & 1 & 27 \\
\hline Guanajuato & 21 & 8 & 3 & 32 \\
\hline Juventino Rosas & 8 & 2 & 1 & 11 \\
\hline Irapuato & 31 & 19 & 5 & 55 \\
\hline León & 85 & 66 & 17 & 168 \\
\hline Slao & 16 & 7 & 2 & 25 \\
\hline Penjamo & 20 & 7 & 1 & 28 \\
\hline Salvatierra & 8 & 4 & 1 & 13 \\
\hline San Jose Iturbide & 10 & 4 & 1 & 15 \\
\hline San Luis de la Paz & 14 & 5 & 2 & 21 \\
\hline Salamanea & 20 & 14 & 4 & 38 \\
\hline Vallagrin & 5 & 3 & 1 & 9 \\
\hline Totales & & & & 619 \\
\hline
\end{tabular}

Fuente: elaboración propia. 
Celaya, Comonfort, San Miguel de Allende, Dolores Hidalgo, San Felipe, Guanajuato, Juventino Rosas, Irapuato, León, Silao, Pénjamo, Salvatierra, San José Iturbide, San Luis de la Paz, Salamanca y Villagrán.

Tabla 3. Muestra de población sector empresarial

\begin{tabular}{|c|c|c|c|c|c|}
\hline \multirow{2}{*}{ Ciudad } & \multicolumn{4}{|c|}{ Sector Empresarial } & \multirow{2}{*}{$\begin{array}{c}\text { Total } \\
\text { Encuestas }\end{array}$} \\
\hline & Industria & Comercio & Servicios & Subtotal & \\
\hline Acámbaro & 1 & 14 & 3 & 18 & 50 \\
\hline Apaseo el Grande & 1 & 6 & 1 & 8 & 31 \\
\hline Celaya & 4 & 68 & 14 & 86 & 214 \\
\hline Comonfort & 1 & 4 & 1 & 6 & 31 \\
\hline San Miguel de Allende & 1 & 1 & 1 & 3 & 63 \\
\hline Dolores Hidalgo & 1 & 12 & 2 & 15 & 67 \\
\hline San Felipe & 1 & 1 & 1 & 3 & 44 \\
\hline Guanajuato & 1 & 20 & 3 & 24 & 81 \\
\hline Juventino Rosas & 1 & 5 & 1 & 7 & 29 \\
\hline Irapuato & 8 & 60 & 19 & 87 & 218 \\
\hline León & 26 & 34 & 24 & 84 & 454 \\
\hline Silao & 1 & 2 & 1 & 4 & 53 \\
\hline Pénjamo & 2 & 8 & 2 & 12 & 59 \\
\hline Salvatierra & 1 & 2 & 1 & 4 & 30 \\
\hline San José Iturbide & 1 & 1 & 0 & 2 & 28 \\
\hline San Luis de la Paz & 0 & 1 & 0 & 1 & 38 \\
\hline Salamanca & 2 & 9 & 3 & 14 & 89 \\
\hline Villagrán & 1 & 6 & 1 & 8 & 26 \\
\hline Totales & & & & 386 & 1605 \\
\hline
\end{tabular}

Fuente: elaboración propia.

La tabla 3 corresponde a la muestra de la población del sector empresarial con participación en total 386 entidades económicas en la entidad federativa de Guanajuato, de las cuales 50 encuestas se aplicaron en Acámbaro a 1 industria, 14 comercios y 3 empresas de servicios; 50 encuestas en Apaseo el Grande a 1 industria, 6 comercios y 1 empresa de servicio; 50 encuestas en Apaseo el Grande a 1 industria, 6 comercios y 1 empresa de servicio; 214 encuestas en Celaya a 4 industrias, 68 comercios y 14 empresas de servicios; 31 encuestas en Comonfort a 1 industria, 4 comercios y 1 empresa de servicio; 63 encuestas en San Miguel de Allende a 1 industria, 1 comercio y 1 empresa de servicio; 67 encuestas en Dolores Hidalgo a 1 industria, 12 comercios y 2 empresas 
de servicios; 44 encuestas en San Felipe a 1 industria, 1 comercio y 1 empresa de servicio; 81 encuestas Guanajuato a 1 industria, 20 comercios y 3 empresas de servicios; 29 encuestas en Juventino Rosas a 1 industria, 5 comercios y 1 empresa de servicio; 218 encuestas en Irapuato a 8 industrias, 60 comercios y 19 empresas de servicios; 454 encuestas se aplicaron en León a 26 industrias, 34 comercios y 24 empresas de servicios; 53 encuestas se aplicaron en Silao a 1 industria, 2 comercios y 1 empresa de servicio; 59 encuestas se aplicaron en Pénjamo a 2 industria, 8 comercios y 2 empresas de servicios; 30 encuestas se aplicaron en Salvatierra a 1 industria, 2 comercios y 1 empresa de servicio; 28 encuestas se aplicaron en San José Iturbide a 1 industria y 1 comercio; 38 encuestas se aplicaron en San Luis de la Paz a 1 comercio; 89 encuestas se aplicaron en Salamanca a 2 industrias, 9 comercios y 3 empresas de servicios; y 26 encuestas se aplicaron en Villagrán a 1 industria, 6 comercios y 1 empresa de servicio.

Se aprecia que del total de submuestras se aplican 1605 encuestas distribuidas de acuerdo al porcentaje de representación proporcional.

Se utiliza el muestreo aleatorio por conglomerados, se divide la población en conglomerados (se agrupa por zonas geográficas u otras áreas de interés para la investigación) y se selecciona aleatoriamente cuáles de ellos formarán parte de la muestra. Una vez seleccionados se toman todos los individuos que componen cada conglomerado (Malhotra 2008).

\section{Paso 6. Plan para el análisis de datos}

Para el análisis de datos se utilizará el software Excel y Dyane V4, que es un programa informático para graficar encuestas.

a. Trabajo de campo o recopilación de datos

Se realizó la logística para el levantamiento de la información en la que fue necesario el apoyo de 83 alumnos.

b. Preparación y análisis de datos

En este apartado se revisaron, codificaron, transcribieron y verificaron cada uno de los cuestionarios para aprobar su correcta aplicación y poder capturarlos en la base de datos.

Resultados. 
Gráfico 1. Las universidades más posicionadas son De Guanajuato, De León, Sabes, La Salle y Politécnica de Guanajuato

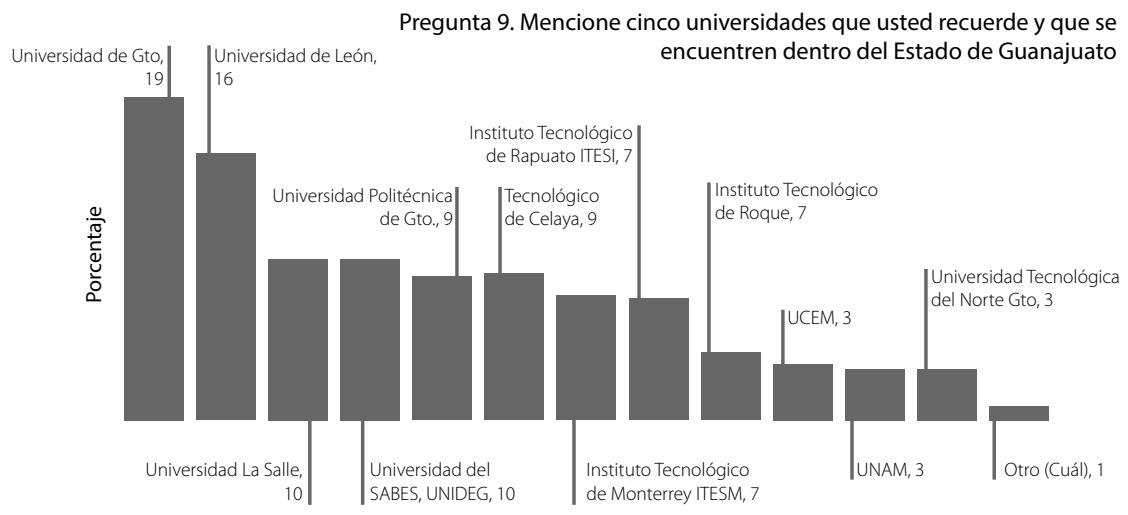

Gráfico 2. Las universidades con mayor prestigio son Instituto Tecnológico de Estudios Superiores de Monterrey, De Guanajuato, De León, Politécnica de Guanajuato y Tecnológico de Celaya

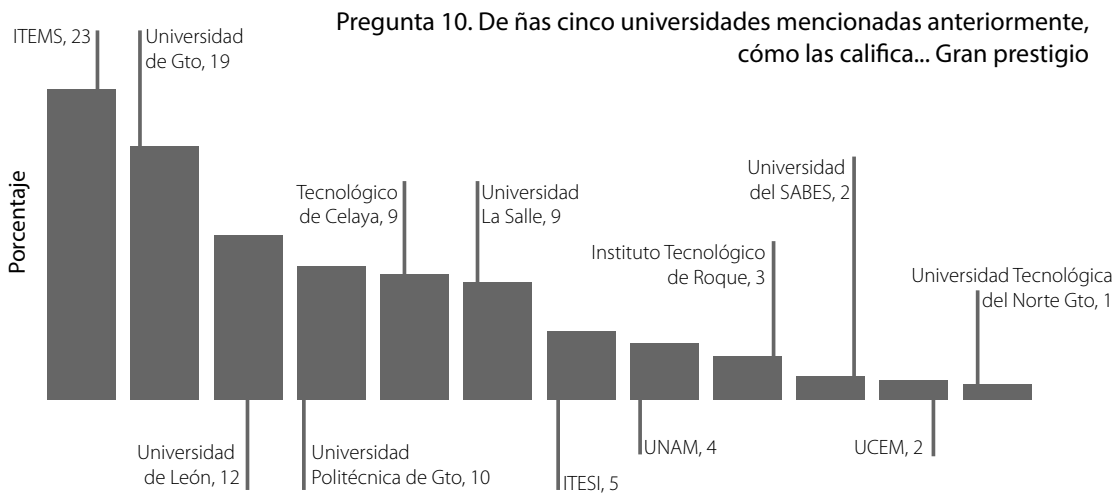


Gráfico 3. Los bachilleratos más posicionados son Colegio Nacional de Educación Profesional Técnica (Conalep), Centro de Bachillerato Tecnológico Industrial y de Servicios, Colegio de Estudios Científicos y Tecnológicos del Estado de Guanajuato (Cecyteg), Sabes y Preparatoria Oficial

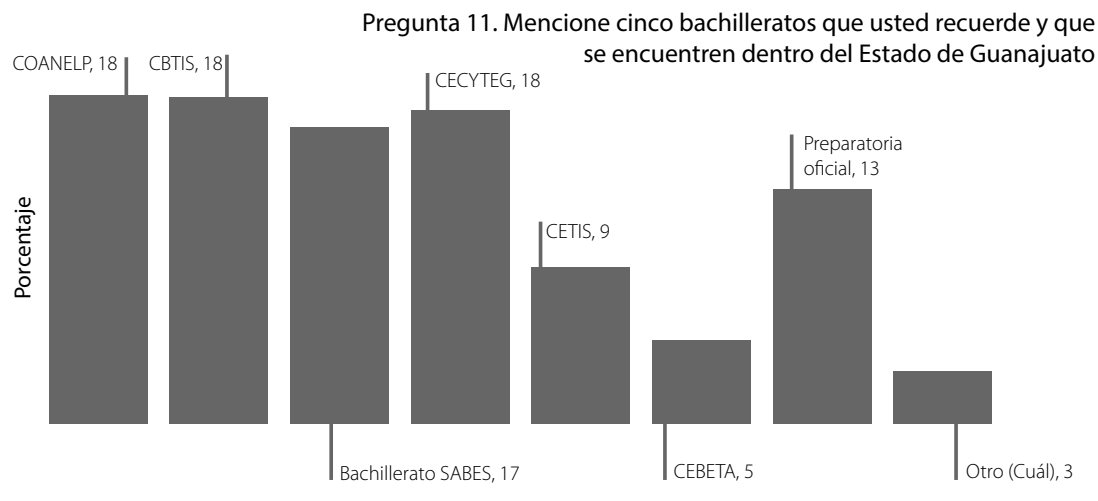

Gráfico 4. Con mayor prestigio: Centro de Bachillerato Tecnológico Industrial y de Servicios, Cecyteg, Conalep, Sabes y Preparatoria Oficial

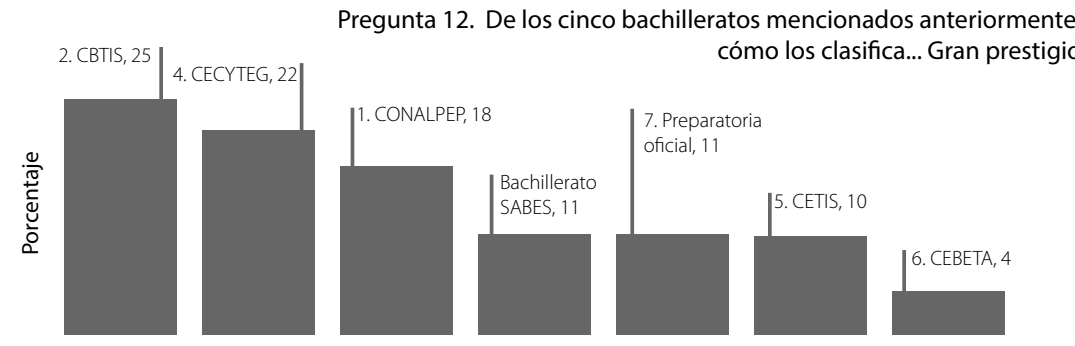

Gráfico 5. Los medios de comunicación más utilizados son televisión, internet, radio y periódico

Pregunta 23. ¿Usted ve t.v.?

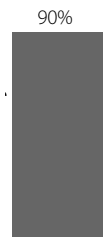

Sí
Pregunta 27. ¿Usted utiliza internet?

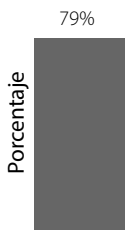

Sí

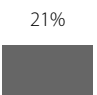

No
Pregunta 14. ¿Usted escucha radio?

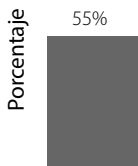

Sí
Pregunta 18 ¿Usted lee algún periódico?

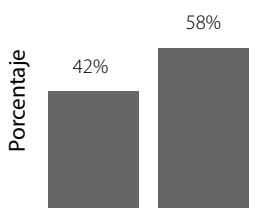

Sí 


\section{Conclusiones}

Los resultados informan la posición y percepción que ocupa cada institución en el mercado, cómo las perciben en costos, calidad, presencia en el estado, criterios necesarios para decidir en qué institución estudiar. Es tarea de las instituciones proponer estrategias y acciones encaminadas a lograr una buena posición en la mente del consumidor.

Es importante mencionar que los datos varían en gran relevancia de acuerdo al análisis de cada una de las muestras, las tres que se han estado mencionando, así como la percepción que existe de un municipio a otro, y la variedad en los hábitos de audiencia.

Por mencionar un ejemplo en la ciudad de Apaseo el Grande, las universidades con mayor posicionamiento, en orden de mención, son: la Universidad de Guanajuato, el Tecnológico de Celaya, la Universidad del Sabes y el Instituto Tecnológico de Roque.

En cambio, en la ciudad de Dolores Hidalgo, las menciones son: Universidad de Guanajuato, Universidad de León, Tecnológico de Monterrey y Universidad La Salle. Y así se puede ir haciendo mención también por municipio y por muestra, dependerá de cómo se prefieran manejar las variables y buscar la relación entre ellas.

En el caso de bachillerato sucede algo similar, como ejemplo se menciona Guanajuato capital, en este caso el bachillerato con mayor posicionamiento es el Centro de Bachillerato Tecnológico Industrial de Servicios, seguido en igual de menciones el Bachillerato Sabes, la Preparatoria Oficial y el Cecyteg.

En el caso de Irapuato, primeramente se menciona al Cecyteg, Conalep, Centro de Bachillerato Tecnológico Industrial y de Servicios, Preparatoria oficial y Bachillerato Sabes.

Se concluye que hoy en día es necesario que las universidades realicen sus estudios de mercado para identificar las características de su mercado potencial, aunque se habla de un estado, en este caso Guanajuato, y que dentro de los dieciocho municipios que se estudian y se comparan existe una gran diversidad y preferencias. Que es necesario estudiarlas, comprender los hábitos que tiene la audiencia, qué medios de comunicación son los que tienen una mayor demanda y cuáles son los más convenientes de acuerdo con la geografía del Estado. 
Este estudio es un referente para la toma de decisiones con una menor incertidumbre para las instituciones, no solamente por la geografía, sino que también es de acuerdo al perfil del consumidor y su percepción de una universidad a otra, de un bachillerato a otro. 


\section{Referencias}

Aristimuño, M., Rodríguez, C. \& Guaita, W. (2011). La responsabilidad social universitaria: indicadores para su evaluación en Instituciones de Educación Superior. Ninth Latin American and Caribbean Conference. 3 y 5 de Agosto 2011. Medellín, Colombia.

Beltrán-Llevador, J., Íñigo-Bajo, E. y Mata-Segreda, A. (2014). La responsabilidad social universitaria, el reto de su construcción permanente. Revista Iberoamericana de Educación Superior, 14(5), 2-16.

Baraibar, E. \& Luna, L. (2012). The influence of transparency of University Social Responsibility in the creation of Reputation. Regional and Sectoral Economics Studies, 12(3), 21-33.

Domínguez, J \& Rama, C. (2012). La Responsabilidad Social Universitaria en la Educación a Distancia. Lima, Perú: Virtual Educa.

Hernández-Arteaga, R., Alvarado-Pérez, J. y Luna, J. (2015). Responsabilidad social en la relación universidad-empresa-estado. Educ. Educ, 18(1), 95 110. doi: $10.5294 /$ edu. 2015.18

Kazmier, L.J. (1998). Estadística aplicada a la administración y a la economía. México: McGraw-Hill.

Kerin, R.A., Hartley S.V. y Redelius, W. (2009). Marketing. México: McGraw-Hill.

Klinger, R., Olaya, J., Rentería, E., Canaval, J., Giraldo, A.L., Mosquera, J. y Bermúdez, M. (2014). Medición de la imagen institucional de una universidad entre estudiantes de último año de educación secundaria. Sophia, 10(1), 83-94.

Lescher, M., Lescher, I. y Caira, N. (2015). Responsabilidad social universitaria en Maracaibo, Venezuela, Telos. Revista de Estudios Interdisciplinarios en Ciencias Sociales, 17(2), 196-207.

Malhotra, N.K. (2008). Investigación de mercados. México: Pearson Prentice Hall.

Martínez-Pichardo, P.J. y Hernández-Oliva, A.V. (2013). Responsabilidad social universitaria: un desafío en la Universidad Pública Mexicana. Contribuciones desde Coatepec, 24, 85-103.

Mehta, R.S. (2011). Corporate Social Responsibility and Universities: Towards an Integrative Approach. International Journal of Social Science and Humanity, 1(4), 1-5. 
Monsiváis, C. (2008). Sobre las universidades públicas. Universidades, LVIII(37), 3-11.

Morales, J.D. (2013). Modelo de Responsabilidad Social Universitaria como factor de formación integral de los ingenieros. Word Engineering Education Forum, 1-10.

Nejati, M., Shafaei, A., Salamzadeh, Y. \& Daraei, M. (2011). Corporate social responsibility and universities: A study of top world universities websites. African Journal of Business Management, 5(2), 440-447.

Ramírez, T. (2010). Estudio de factibilidad, elemento clave para la apertura de un programa educativo pertinente en la región suroeste del Estado de Guanajuato. Cuadernos de Educación y Desarrollo, 2(13).

Ramos, C., García, J. y Pérez, Y. (2011). La Universidad de servicio como paradigma de la Educación Superior. Revista de Ciencias Sociales, XVII(1), 48-58.

Reinhard, K., Osburg, T. \& Townsend, R. (2010). Introducing corporate social responsibility as component in cooperative education: Results from a student research Project in Germany and The United States supported by Intel Corporation. Asia-Pacific Journal of Cooperative Education, 11(1), 1-12.

Rivera, M.J., \& Martínez, M.Y. (2014). Diagnóstico de la imagen y posicionamiento de la Universidad Nacional Abierta y a Distancia-Garagoa en el sector productivo de la región del Valle de Tenza-Boyacá. (Tesis de licenciatura). Universidad Nacional Autónoma de México. Recuperado de repository.unad.edu.co/bitstream/10596/3393/1/33677203.pdf

Stanton, W.J., Etzel, M.J. y Walker, B. J. (2007). Fundamentos de marketing. México. McGraw-Hill.

Thöni, E. \& Schneller, C. (2011). Knowledge Societies: Universities and their Social Responsabilities. Asia-Europe Foundation's Eduaction Hub and the University of Innsbruck, 1-76.

Valarezo, K. \& Tuñez, J.M. (2014). Responsabilidad Social Universitaria. Revista de Comunicación, 13, 84-117.

Vasilescu, R., Barna, C., Epure, M. \& Baicu, C. (2010). Developing University social responsibility: A model for the challenges of the new civil society. Procedia Social and Behavioral Sciences, 2, 4177-4182.

Zeithmal, V.A., Bitner, M. J. \& Gremler, D.D. (2009). Marketing de servicios. México: McGraw-Hill. 